\title{
Transition to Instability of Planar Viscous Shock Fronts: the Refined Stability Condition
}

\author{
Sylvie Benzoni-Gavage, Denis Serre, and Kevin Zumbrun
}

\begin{abstract}
Classical inviscid stability analysis determines stability of shock waves only up to a region of neutral stability occupying an open set of physical parameters. To locate a precise transition point within this region, it has been variously suggested that nonlinear and or viscous effects should be taken into account. Recently, Zumbrun and Serre showed that transition under localized $\left(L^{1} \cap H^{s}\right)$ perturbations is in fact entirely decided by viscous effects, and gave an abstract criterion for transition in terms of an effective viscosity coefficient $\beta$ determined by second derivatives of the Evans function associated with the linearized operator about the wave. Here, generalizing earlier results of Kapitula, Bertozzi et al, and Benzoni-Gavage et al., we develop a simplified perturbation formula for $\beta$, applicable to general shock waves, that is convenient for numerical and analytical investigation.
\end{abstract}

Keywords. Evans function, viscous conservation laws, spectral stability

Mathematics Subject Classification (2000). Primary 35P15, secondary 35B40, $47 \mathrm{~A} 75$

S. Benzoni-Gavage: Institut Camille Jordan (UMR CNRS-UCBL \# 5208), Université Claude Bernard Lyon 1, France; benzoni@math.univ-lyon1.fr

Partially supported by TMR project "Hyperbolic conservation laws", contract \# ERB FMRX-CT96-0033.

D. Serre: Unité de Mathématiques Pures et Appliquées (UMR CNRS-ENSL \# 5669), Ecole Normale Supérieure de Lyon, France; serre@umpa.ens-lyon.fr

Partially supported by TMR project "Hyperbolic conservation laws", contract \# ERB FMRX-CT96-0033.

K. Zumbrun: Department of Mathematics, Indiana University, Bloomington IN, USA; kzumbrun@indiana.edu

Partially supported by NSF grants number DMS-9107990 and DMS-0300487. K.S. thanks the Ecole Normale Superieure, Lyon, for their hospitality during the visit in which this work was initiated. 


\section{Introduction}

Consider a planar shock solution

$$
u(x, t)=\bar{u}\left(\frac{x_{1}-s t}{\nu}\right), \quad \lim _{z \rightarrow \pm \infty} \bar{u}(z)=u_{ \pm},
$$

of a general system of viscous conservation laws

$$
u_{t}+\sum_{j} f^{j}(u)_{x_{j}}=\nu \sum_{j, k}\left(B^{j k}(u) u_{x_{k}}\right)_{x_{j}}
$$

$u, f^{j} \in \mathbb{R}^{n}, B \in \mathbb{R}^{n \times n}, x \in \mathbb{R}^{d}, \nu>0$, satisfying the traveling-wave ODE

$$
B^{11}(\bar{u}) \bar{u}^{\prime}=f^{1}(\bar{u})-f^{1}\left(u_{-}\right)-s\left(\bar{u}-u_{-}\right) .
$$

Solution (1.1) converges as $\nu \rightarrow 0$ to an ideal shock solution

$$
\begin{gathered}
u(x, t)= \begin{cases}u_{-} & x<s t \\
u_{+} & x \geq s t,\end{cases} \\
f^{1}\left(u_{+}\right)-f^{1}\left(u_{-}\right)-s\left(u_{+}-u_{-}\right)=0,
\end{gathered}
$$

of the corresponding inviscid system

$$
u_{t}+\sum_{j} f^{j}(u)_{x_{j}}=0
$$

For definiteness, we restrict to the case of a classical Lax-type shock (defined Section 2). Equivalently (see [62,67] for strictly parabolic systems, [48,63] for real viscosity systems), we assume that $\bar{u}$ is a unique, transverse orbit solving the connection problem (1.3), connecting nonhyperbolic rest points $u_{+}$and $u_{-}$. Nonclassical under- or overcompressive shocks may be treated similarly; see, e.g., the treatment of undercompressive phase transitions in [3].

We have in mind the physical example in which (1.1) represents a gasdynamical shock wave and (1.2) and (1.6) the compressible Navier-Stokes and Euler equations, respectively. Gas dynamical shocks are under normal circumstances quite stable; however, in extreme parameter ranges they are known to become unstable [2]. Natural questions, therefore, are the stability under perturbation of viscous vs. ideal shocks as solutions of their respective systems, and the predictive value of these mathematical considerations in determining physically observed transitions to instability.

Inviscid stability analysis centers about the Lopatinski determinant

$$
\Delta(\tilde{\xi}, \lambda):=\operatorname{det}\left(\begin{array}{lllllll}
\mathcal{R}_{1}^{-} & \cdots & \mathcal{R}_{p-1}^{-} & \mathcal{R}_{p+1}^{+} & \cdots & \mathcal{R}_{n}^{+} & \left.\lambda[u]+i\left[f^{\tilde{\xi}}\right]\right)
\end{array}\right.
$$


$\tilde{\xi}=\left(\xi_{2}, \ldots, \xi_{d}\right) \in \mathbb{R}^{d-1}, \lambda=\gamma+i \tau \in \mathbb{C}, \tau>0$, a spectral determinant whose zeroes correspond to normal modes $e^{\lambda t} e^{i \tilde{\xi} \cdot \tilde{x}} w\left(x_{1}\right), \tilde{x}=\left(x_{2}, \ldots, x_{d}\right)$, of the constant-coefficient linearized equations about (1.4), or spectra of the linearized operator about the wave. Here $f^{\tilde{\xi}}:=\sum_{j=2}^{d} \xi_{j} f^{j}$ and $\left\{\mathcal{R}_{p+1}^{+}, \ldots, \mathcal{R}_{n}^{+}\right\}$ and $\left\{\mathcal{R}_{1}^{-}, \ldots, \mathcal{R}_{p-1}^{-}\right\}$denote bases for the unstable/resp. stable subspaces of

$$
\mathcal{A}_{+}(\tilde{\xi}, \lambda):=\left(\lambda I+i d f^{\tilde{\xi}}\left(u_{ \pm}\right)\right)\left(d f^{1}\left(u_{ \pm}\right)\right)^{-1} .
$$

Weak stability $|\Delta|>0$ is clearly necessary for linearized stability, while strong, or uniform stability, $\frac{|\Delta|}{|(\tilde{\xi}, \lambda)|} \geq c_{0}>0$, is sufficient for nonlinear stability. Between strong instability, or failure of weak stability, and strong stability, there lies a region of neutral stability corresponding to the appearance of surface waves propagating along the shock front. For details, see, e.g., $[4,42-44,49,56-58,62-$ 64,67 , and references therein ${ }^{1}$; for generalizations of (1.7) to the nonclassical and or nonconservative case, see $[11,18,27,39,52,61,67]$.

The region of neutral inviscid stability typically occupies an open set in physical parameter space $[4,42-44,62,63]$, and thus the basic inviscid theory we have just described does not identify a precise transition point from stability to instability as shock parameters are varied. It has been suggested [2] that accounting of additional nonlinear and or second-order transport effects might resolve this issue. On the other hand, it is noted also that, at the high energy levels needed to observe instability, experiments are somewhat inconclusive, with onset of instability sometimes appearing to occur earlier, within the (mathematical) strongly stable region. Barmin and Egorushkin [2] speculate that the latter might be a result of incomplete modeling of phase-transitional effects associated with ionization.

Weakly nonlinear analysis within the inviscid framework ${ }^{2}$ has been pursued by Majda and Rosales in the related context of detonation waves, with interesting asymptotic and numerical results; see $[46,47]$. We focus here on a different approach initiated by Zumbrun and Serre [22,62-64,67] within the context of the viscous equations (1.2), incorporating nonlinear and viscous effects, concerning long-time stability with respect to localized $\left(L^{1} \cap H^{s}\right)$ perturbations.

Remark 1.1. As discussed in [23,63], inviscid stability theory concerns shorttime stability, or well-posedness (automatic for parabolic equations) with respect to $H^{s}$ perturbations, whereas standard viscous stability theory concerns long-time asymptotic stability with respect to the more restrictive class of $L^{1} \cap H^{s}$ perturbations. These notions are loosely related by rescaling, but in

\footnotetext{
${ }^{1}$ See especially the seminal work [13].

${ }^{2}$ More precisely, for the Zeldovich-von Neumann-Doering (ZND) model including reaction but not transport effects; as discussed in [31,62], this model is intermediate to the ChapmanJouget and reactive Navier-Stokes models analogous to (1.6) and (1.2).
} 
general give complementary information; see discussion [23]. For a short-time viscous analysis in the spirit of [42-44], see the treatment of the inviscid limit in $[23-25]$.

Viscous stability analysis centers about the Evans function $D(\tilde{\xi}, \lambda), \tilde{\xi}=$ $\left(\xi_{2}, \ldots, \xi_{d}\right) \in \mathbb{R}^{d-1}, \lambda=\gamma+i \tau \in \mathbb{C}, \tau>0$, a spectral determinant analogous to the Lopatinski determinant of the inviscid theory, whose zeroes correspond to normal modes $e^{\lambda t} e^{i \tilde{\xi} \cdot \tilde{x}} w\left(x_{1}\right), \tilde{x}=\left(x_{2}, \ldots, x_{d}\right)$, of the linearized equations about (1.1) (now variable-coefficient), or spectra of the linearized operator about the wave. The main result of [67], establishing a rigorous relation between viscous and inviscid stability, was the asymptotic expansion

$$
D(\tilde{\xi}, \lambda)=\gamma \Delta(\tilde{\xi}, \lambda)+o(|(\tilde{\xi}, \lambda)|)
$$

of $D$ about the origin $(\tilde{\xi}, \lambda)=(0,0)$, where $\gamma$ is a constant measuring tranversality of (1.1) as a connecting orbit of (1.3). Equivalently, considering $D(\xi, \lambda)=D\left(\rho \xi_{0}, \rho \lambda_{0}\right)$ as a function of polar coordinates $\left(\rho, \xi_{0}, \lambda_{0}\right)$, we have

$$
\left.D\right|_{\rho=0}=0 \quad \text { and }\left.\quad \frac{\partial}{\partial \rho}\right|_{\rho=0} D=\gamma \Delta\left(\xi_{0}, \lambda_{0}\right) .
$$

An important consequence of (1.9) is that weak inviscid stability, $|\Delta|>0$, is necessary for weak viscous stability, $|D|>0$ (an evident necessary condition for linearized viscous stability). For, (1.9) implies that the zero set of $D$ is tangent at the origin to the cone $\{\Delta=0\}$ (recall, (1.7), that $\Delta$ is homogeneous, degree one), hence enters $\{\tau>0\}$ if $\{\Delta=0\}$ does. Moreover, in case of neutral inviscid stability $\Delta\left(\xi_{0}, i \tau_{0}\right)=0,\left(\xi_{0}, i \tau_{0}\right) \neq(0,0)$, one may extract a further, refined stability condition

$$
\beta:=-\left.\frac{D_{\rho \rho}}{D_{\rho \lambda}}\right|_{\rho=0} \geq 0
$$

necessary for weak viscous stability. For, (1.10) then implies

$$
\left.D_{\rho}\right|_{\rho=0}=\gamma \Delta\left(\xi_{0}, i \tau_{0}\right)=0
$$

whence Taylor expansion of $D$ yields that the zero level set of $D$ is concave or convex toward $\tau>0$ according as the sign of $\beta$; see [67] for details. As discussed in $[62,67]$, the constant $\beta$ has a heuristic interpretation as an effective diffusion coefficient for surface waves moving along the front.

Under additional structural assumptions, satisfied in particular for the equations of gas dynamics, converse results have been established in [62-64], showing that $\beta>0$ is sufficient for "low-frequency linearized stability" in the sense that this condition, together with "high-frequency stability" $|D(\xi, \lambda)>0|$ for 
$|(\xi, \lambda)| \geq c_{0}>0$ and $\tau \geq 0$, is sufficient for linearized and nonlinear stability. This means that, in the context of long-time viscous stability with respect to localized perturbations, transition to instability is in principle determined under existing theory.

For, taking a fixed left-state $u_{-}$and moving along the Hugoniot curve of right states $u_{+}$satisfying the Rankine-Hugoniot relation (1.5), we find that the associated shock profile transitions from low-frequency stability to lowfrequency instability either at the endpoints of the region of neutral inviscid stability (readily computable; see, e.g., $[4,13,63]$ ) or at a point $\beta=0$ where $\beta$ change sign. Likewise, transition from high-frequency stability to highfrequency instability occurs at (generically isolated) points along the Hugoniot curve at which high-frequency eigenvalues cross the imaginary axis. In either case, transition must occur at one of a discrete set of points.

Thus, we have the following situation. Recalling that inviscid theory is basically a low-frequency, or long-wave approximation neglecting inner shock structure, we see that, insofar as inviscid theory is predictive of stability, a refinement quantifying the transition to instability may be obtained by evaluation of $\beta$ in (1.11).

On the other hand, it may well be that, in certain circumstances, stability is determined, rather, by high-frequency, viscous effects completely foreign to inviscid analysis; indeed, this, rather than unmodeled phase-transitional effects, might be an explanation for experimentally observed occasional early onset of instability as described in [2]. To detect the latter situation, we see no option other than a fully resolved numerical evaluation of the Evans function as discussed, e.g., in $[7-10,30]$.

Remark 1.2. In the related context of detonation waves, experimental and theoretical evidence indicates that transition to instability typically occurs at high frequency, corresponding to a relative Poincaré-Hopf bifurcation, or complex conjugate pair of eigenvalues crossing the imaginary axis; see, e.g., [31,40, $41,59,60]$, and references therein ${ }^{3}$. Whether and when high-frequency transition occurs for shock waves remains an important philosophical question, possibly connected with existence of an increasing thermodynamic entropy; see [63, 1.20.2].

This state of affairs suggests a two-pronged approach to determination of stability transitions, on one hand focusing on low-frequency stability and determination of $\beta$, and on the other (in more limited situations) on high-frequency

\footnotetext{
${ }^{3}$ In particular, an expansion (1.9) relates the Evans function $D$ for the full, reactive NavierStokes equations to the Lopatinski determinant $\Delta$ for the simplified Chapman-Jouget model, with $\Delta$ strongly stable for an ideal gas despite experimentally observed instability in some regimes [31].
} 
stability and full numerical evaluation of the Evans function, the expectation being that low-frequency stability "usually" determines transition, with infrequent (if any) exceptions in unusual parameter regimes. In this paper, we initiate the first (and expectedly the primary one) of these directions of investigation by deriving a compact, Lyapunov-Schmidt-type formula for $\beta$ that is convenient for numerical and analytical investigation. We intend to use this formula in followup work to investigate low-frequency transition to instability in various physically and mathematically interesting cases.

Specifically, we establish the following result generalizing (1.9).

Theorem 1.3. Let $\bar{u}$ be a Lax shock and $\tilde{\xi} \in \mathbb{R}^{d-1}, \tau \in \mathbb{R}$ such that $\Delta(\tilde{\xi}, i \tau)=0$, satisfying $(\mathrm{H} 0)-(\mathrm{H} 7)$ (defined below). Then, $\beta:=-\frac{D_{\rho \rho}}{D_{\rho \lambda}}(\tilde{\xi}, i \tau)$ is given by

$$
\begin{aligned}
\beta & =-\Delta_{\lambda}(\tilde{\xi}, \tau)^{-1}\left[\left\langle l,-2\left[\frac{\partial}{\partial \rho} L_{\rho}\right]_{\left.\right|_{\rho=0}} \tilde{y}-\left[\left(\frac{\partial}{\partial \rho}\right)^{2} L_{\rho}\right]_{\left.\right|_{\rho=0}} \bar{u}^{\prime}\right\rangle+\mathcal{B}\right] \\
& =-\Delta_{\lambda}(\tilde{\xi}, \tau)^{-1}\left[\left\langle l, 2\left(\lambda I+A^{\tilde{\xi}}-i B^{1 \tilde{\xi}} \partial_{x_{1}}-i \partial_{x_{1}} B^{\tilde{\xi} 1}\right) \tilde{y}+2 B^{\tilde{\xi} \tilde{\xi}} \bar{u}^{\prime}\right\rangle+\mathcal{B}\right]
\end{aligned}
$$

where $\Delta(\cdot, \cdot)$ is as defined in $(1.7),\langle\cdot, \cdot\rangle$ denotes the standard complex $L^{2}$ inner product, $l$ is the constant vector determined by the exterior product

$$
l:=\mathcal{R}_{1}^{-} \wedge \cdots \wedge \mathcal{R}_{p-1}^{-} \wedge \mathcal{R}_{p+1}^{+} \wedge \cdots \wedge \mathcal{R}_{n}^{+},
$$

$\tilde{y}$ is the (unique, modulo $\left.\bar{u}^{\prime}\right)$ solution of the (first order) variational equation $L_{0} \tilde{y}=-\left[\frac{\partial}{\partial \rho} L_{\rho}\right]_{\left.\right|_{\rho=0}} \bar{u}^{\prime}$, or

$$
\partial_{x_{1}}\left(B^{11} \partial_{x_{1}}-A^{1}\right) \tilde{y}=\left(\lambda I+A^{\tilde{\xi}}-i B^{1 \tilde{\xi}} \partial_{x_{1}}-i \partial_{x_{1}} B^{\tilde{\xi} 1}\right) \bar{u}^{\prime}
$$

satisfying boundary conditions

$$
\begin{aligned}
& \tilde{y}(+\infty) \in\left(A_{+}^{1}\right)^{-1} \operatorname{Span}\left\{\mathcal{R}_{p+1}^{+}, \ldots, \mathcal{R}_{n}^{+}\right\} \\
& \tilde{y}(-\infty) \in\left(A_{-}^{1}\right)^{-1} \operatorname{Span}\left\{\mathcal{R}_{1}^{-}, \ldots, \mathcal{R}_{p-1}^{-}\right\}
\end{aligned}
$$

and $\mathcal{B}$ is a boundary term described in (3.10) of Proposition 3.3 below. (Here, $L_{\rho}=L_{\rho}(\tilde{\xi}, \lambda)$ is the Fourier-Laplace transform of the linearized operator about the wave, written in polar coordinates $(\rho, \tilde{\xi}, \lambda)$, $\rho$ the radius; see (3.2).)

Remark 1.4. Noting that $\Delta$ can be expressed as

$$
\Delta=\left\langle l,\left(\lambda I+A^{\tilde{\xi}}-i B^{1 \tilde{\xi}} \partial_{x}-i \partial_{x} B^{\tilde{\xi} 1}\right) \bar{u}^{\prime}\right\rangle,
$$

(see related calculations $(2.26)-(2.29)$ of [67]) we find that formula (1.12) is indeed independent of the choice of $\tilde{y}$, since $\Delta=0$ by assumption. 
For gas dynamics, $\mathcal{R}_{j}^{ \pm}$and $\Delta$, hence $\mathcal{B}$ and $\Delta_{\lambda}$ are explicitly computable (see [62, Appendix $\mathrm{C}]$, or $[4,13]$ ), hence evaluation of $\beta$ reduces to solution of (1.14) for $\tilde{y}$. More generally, $\mathcal{B}$ and $\Delta_{\lambda}$ may be approximated numerically in well-conditioned fashion. Numerical approximation of $\tilde{y}$ is also a wellconditioned problem, as discussed in Section 4. Thus, Theorem 1.3 indeed gives a prescription for the desired efficient determination of $\beta$, and thereby of the transition point between stability and instability of viscous shock waves.

Remark 1.5. The vector $l$ can be recognized as an "effective" adjoint eigenfunction dual to $\bar{u}^{\prime}$, see discussion $[62,66]$. This establishes the connection with Lyapunov-Schmidt decomposition and the generalized Melnikov formulae of [32]. Specifically, our formula may be seen to agree, up to boundary term $\mathcal{B}$, with those of [32], generalizing one-dimensional results of $[5,6,65]$. As discussed in $[5,65]$, the appearance of a boundary term is associated with the absence of spectral gap for the linearized operator $L_{0}$. Similar boundary terms have been observed in [33-35] in the context of perturbed nonlinear Schrödinger equations. Related second-derivative calculations may be found in [38].

Plan of the paper. In Section 2, we recall the construction of the Evans and Lopatinski determinants and other background facts. In Section 3, we carry out the proof of (3.1) and Theorem 1.3. Finally, in Section 4, we discuss numerical approximation of the function $\tilde{y}$ appearing in (1.12).

Note. After the completion of this article, we have learned of new inviscid results of Coulombel and Secchi [12], which include among other things the conclusion that inviscid shocks in the "neutral stability" region are in fact nonlinearly stable in the sense of Majda. This important work answers in the positive the longstanding mathematical question of inviscid stability in the neutral regime. However, it leaves open the fundamental physical question whether inviscid well-posedness is a useful criterion to detect transition to instability, lending additional interest to our investigations.

\section{Preliminaries}

For clarity of exposition, we carry out the analysis in the simpler, strictly parabolic case. It is a routine but tedious exercise to verify that all calculations carry over to the case of "real", or physical, partially parabolic viscosity treated in $[62,64]$ under the standard hypotheses therein: in particular, to the Navier-Stokes equations of compressible gas- or magnetohydrodynamics (MHD). See [62], Sections 3.1-3.2 for the analog of Proposition 3.1; the calculation of $\beta$ goes similarly. 
2.1. Assumptions. We make the standard hypotheses ( $[21,63,66,67])$ :

(H0) $f^{j}, B^{j k} \in C^{2}$.

(H1) $\Re \sigma\left(\sum_{j, k} \xi_{k} \xi_{j} B^{j k}(\bar{u}(\cdot))\right)>0$ for all nonzero $\xi \in \mathbb{R}^{d}$.

(H2) $\sigma\left(\sum_{j} D f^{j}\left(u_{ \pm}\right) \xi_{j}\right)$ real, semi-simple for all $\xi \in \mathbb{R}^{d}, s \notin \sigma\left(D f^{1}\left(u_{ \pm}\right)\right)$.

(H3) $\Re \sigma\left(-i \sum_{j} D f\left(u_{ \pm}\right) \xi_{j}-\sum_{j, k} B^{j k}\left(u_{ \pm}\right) \xi_{k} \xi_{j}\right) \leq-\theta|\xi|^{2}, \theta>0$.

(H4) The orbit $\bar{u}(\cdot)$ is a (unique) transverse connection for (1.3) between nonhyperbolic rest points $u_{ \pm}$.

Hypotheses (H0)-(H3) correspond, respectively, to regularity, (local) parabolicity, hyperbolicity of $u_{ \pm} /$nonsonicity of the shock triple $\left(u_{-}, u_{+}, s\right)$, and (strong) $L^{2}$ linearized stability of the constant solutions $u \equiv u_{ \pm}$. Hypothesis (H4) includes the information that the dimensions of the unstable manifold of $u_{-}$and the stable manifold of $u_{+}$, i.e. (see (1.3)), the unstable subspace of $\left(B^{11}\right)^{-1}\left(d f^{1}\left(u_{-}\right)-s I\right)$ and the stable subspace of $\left(B^{11}\right)^{-1}\left(d f^{1}\left(u_{+}\right)-s I\right)$ sum to $n+1$. By a lemma of [45] (see [62,67]), this together with (H2)-(H3) implies that the dimensions $i_{-}$and $i_{+}$of the unstable subspace of $d f^{1}\left(u_{-}\right)-s I$ and the stable subspace of $d f^{1}\left(u_{+}\right)-s I$ sum also to $n+1$, or, in the language of hyperbolic theory, there are $n+1$ total characteristics incoming to the shock. The number of incoming characteristics defines (1.4) as a classical, Lax p-shock, $p=n-i_{-} ;$see $[62,67]$ for further discussion.

Remark 2.1. Hypothesis (H4) specializes to the Lax case and slightly strenthens the the more general "weak transversality" hypothesis of $[62,67]$ that, local to $\bar{u}(\cdot)$, the set of solutions of (1.1)-(1.3) connecting the same endstates $u_{ \pm}$with same speed $s$ form a smooth manifold $\left\{\bar{u}^{\delta}\right\}, \delta \in U \subset \mathbb{R}^{\ell}$. In the present case, $\left\{\bar{u}^{\delta}\right\}=\{\bar{u}(\cdot-\delta)\}$ and $\ell=1$. For more general, nonclassical over- and undercompressive shocks (discussed in detail in $[62,67]$ ), the number of incoming characteristics and the value of $\ell$ are essentially arbitrary.

2.2. The Evans function. Loosely following [67], we now briefly recall the construction of the Evans function. Without loss of generality, take $s=0$, so that $\bar{u}\left(x_{1}\right)$ is a standing-wave solution. Linearizing (1.2) about $\bar{u}(\cdot)$, we obtain

$$
v_{t}=L v:=\sum\left(B^{j k} v_{x_{k}}\right)_{x_{j}}-\sum\left(A^{j} v\right)_{x_{j}}
$$

where coefficients

$$
B^{j k}:=B^{j k}(\bar{u}), \quad A^{j} v:=D f^{j}(\bar{u}) v-D B^{j 1}(\bar{u})\left(v, \bar{u}_{x_{1}}\right)
$$

are smooth functions of $x_{1}$ alone.

Seeking normal modes $v(x, t)=e^{\lambda t} e^{i \tilde{\xi} \cdot x} w\left(x_{1}\right)$ of $(2.1), w \in L^{2}\left(x_{1}\right)$ (equivalently, taking the Fourier-Laplace transform in transverse spatial directions 
$\tilde{x}=\left(x_{2}, \ldots, x_{n}\right)$ and time $\left.t\right)$, we are led to the family of generalized eigenvalue equations

$$
\begin{aligned}
0= & \left(L_{\tilde{\xi}}-\lambda\right) w \\
= & \left(B^{11} w^{\prime}\right)^{\prime}-\left(A^{1} w\right)^{\prime}+i \sum_{j \neq 1} B^{j 1} \xi_{j} w^{\prime}+i\left(\sum_{k \neq 1} B^{1 k} \xi_{k} w\right)^{\prime} \\
& -i \sum_{j \neq 1} A^{j} \xi_{j} w-\sum_{j \neq 1, k \neq 1} B^{j k} \xi_{j} \xi_{k} w-\lambda w,
\end{aligned}
$$

where "'," denotes $\frac{\partial}{\partial x_{1}}$. Evidently, existence of solutions corresponds to linear dependency between the unstable manifold at $x_{1} \rightarrow-\infty$ and the stable manifold at $x_{1} \rightarrow+\infty$ of solutions of (2.3).

Lemma 2.2. There exist choices $w_{j}^{ \pm}\left(\tilde{\xi}, \lambda, x_{1}\right)$ of bases

$$
\left\{w_{1}^{+}, \ldots, w_{2}^{+}\right\} \quad \text { and } \quad\left\{w_{n+1}^{-}, \ldots, w_{2 n}^{-}\right\}
$$

spanning the stable/unstable manifolds at $-\infty /+\infty$ of solutions of $(2.3)$, jointly analytic in $(\tilde{\xi}, \lambda)$ in a neighborhood of any $(\tilde{\xi}, \lambda) \in \mathbb{R}^{d-1} \times\{\Re \lambda>0\}$.

Proof. It is sufficient to show the corresponding result for the limiting, constantcoefficient equations as $x_{1} \rightarrow \pm \infty$, the variable-coefficient result then following by asymptotic ODE theory: specifically, the "conjugation lemma" of [50] (a generalization of the "gap lemma" of $[21,34]$ ), asserting as a consequence of exponential convergence of the coefficients that there is an $2 n \times 2 n$ bounded invertible change of coordinates $Q(\tilde{\xi}, \lambda, x)$ on $x>0$ (resp. $<0$ ), locally analytic in $(\tilde{\xi}, \lambda)$, taking solutions $\left(\hat{w}, \hat{w}^{\prime}\right)$ of the limiting constant-coefficient equations to solutions $\left(w, w^{\prime}\right)=Q\left(\hat{w}, \hat{w}^{\prime}\right)$ of the variable-coefficient equations. But, the result for the constant-coefficient equations follows by spectral separation of stable and unstable eigenspaces on $\Re \lambda>0$, an easy consequence of (H3). For details, see [67].

Definition 2.3. The (local) Evans function $D(\tilde{\xi}, \lambda)$ associated with $(2.3)$ is defined as the Wronskian

$$
D(\tilde{\xi}, \lambda):=\operatorname{det}\left(\begin{array}{llllll}
w_{1}^{+} & \cdots & w_{n}^{+} & w_{n+1}^{-}, & \cdots & w_{2 n}^{-} \\
w_{1}^{+\prime} & \cdots & w_{n}^{+\prime} & w_{n+1}^{-} & \cdots & w_{2 n}^{-\prime}
\end{array}\right)_{\left.\right|_{x_{1}=0}} .
$$

Evidently, $D(\cdot, \cdot)$ is jointly analytic in $(\tilde{\xi}, \lambda)$ on its domain of definition. The Evans function as usual is specified only up to a nonvanishing analytic multiplier, since the functions $w_{j}^{ \pm}$are specified only up to analytic change of basis. 
Remark 2.4. The Evans function may alternatively be defined globally as a $C^{\infty}$ function in $(\tilde{\xi}, \lambda)[25]$, and in the one-dimensional case globally analytic in $\lambda$ [21]. Similarly, using homogeneity to reduce to a single complex argument, the Lopatinski determinant may be chosen globally $C^{\infty}$, and globally analytic in the two-dimensional (more generally, the rotationally symmetric) case ${ }^{4}$. Local $C^{\infty}$ regularity is sufficient for the multidimensional theory of [22-25, 62-64,67].

We now introduce the main object of our attention, the "radial Evans function"

$$
D_{\tilde{\xi}, \lambda}(\rho):=D(\rho \tilde{\xi}, \rho \lambda), \quad \rho \in(0,+\infty)
$$

This function is evidently jointly analytic in $(\tilde{\xi}, \lambda, \rho)$ where it is defined. The key point is to understand behavior as $\tau, \rho \rightarrow 0^{+}$(recall, $\tau:=\Re \lambda$ ).

2.3. Variable multiplicity and glancing sets. Denote by $a_{j}^{ \pm}(\xi), j=1, \ldots, n$ the eigenvalues (real by $(\mathrm{H} 2)$ ) of hyperbolic initial-value symbol $\sum_{j=1}^{d} A_{ \pm}^{j} \xi_{j}$, indexed by increasing order, without loss of generality chosen to be (real) homogeneous degree one. Define $\Xi_{ \pm}^{r}$ to be the sets of $\xi$ at which $a_{r}^{ \pm}$changes multiplicity. Away from $\Xi_{ \pm}^{r}$, each $a_{ \pm}^{r}$ is analytic in $\xi$. Define $\Theta_{ \pm}^{r}$ to be the sets of $\xi \notin\left(\cup \Xi^{r}\right)_{ \pm}$at which $\frac{\partial a_{r}^{ \pm}}{\partial \xi_{1}}=0$.

Definition 2.5. Setting $\xi=\left(\xi_{1}, \ldots, \xi_{d}\right)=$ : $\left(\xi_{1}, \tilde{\xi}\right)$, we define the variable multiplicity set $\mathcal{V}$ as the collection of all $(\tilde{\xi}, i \tau)$ such that, for some real $\xi_{1}$ and $1 \leq r \leq n, \tau=-a_{r}^{ \pm}\left(\xi_{1}, \tilde{\xi}\right)$ and $\left(\xi_{1}, \tilde{\xi}\right) \in \Xi_{ \pm}^{r}$. Similarly, we define the glancing set $\mathcal{G}$ as the collection of all $(\tilde{\xi}, i \tau)$ such that, for some real $\xi_{1}$ and $1 \leq r \leq n$, $\tau=-a_{r}^{ \pm}\left(\xi_{1}, \tilde{\xi}\right),\left(\xi_{1}, \tilde{\xi}\right) \in \Theta_{ \pm}^{r}$. These are codimension one (hence measure zero) in $\mathbb{R}^{d-1} \times\{\Re \lambda=0\}$, as the union of the projections onto $(\tilde{\xi}, i \tau)$ of the graphs $\left(\xi,-i a_{r}^{ \pm}(\xi)\right)$ over the codimension one sets $\Xi_{ \pm}^{r}, \Theta_{ \pm}^{r}$.

The glancing set consists of points at which the analytic (temporal) modes $i \tau=-i a_{r}^{ \pm}(\xi)$ of the hyperbolic initial-value symbol $\sum_{j} i \xi_{j} A_{ \pm}^{j}$ are not analytically invertible as functions $i \xi_{1}=\alpha_{r}(\tilde{\xi}, \tau), r=1, \ldots, n$, describing the (spatial) modes of the hyperbolic initial-boundary-value symbol $-\left(A_{ \pm}^{1}\right)^{-1}(i \tau+$ $\left.\sum_{j=2}^{d} i \xi_{j} A_{ \pm}^{j}\right)$. The two symbols are linked by the full Fourier transform $(i \tau+$ $\left.\sum_{j} i \xi_{j} A_{ \pm}^{j}\right) \hat{v}=0$ of the frozen-coefficient linearized hyperbolic problem.

Remark 2.6. For gas dynamics, $\mathcal{V}$ is empty and $\mathcal{G}$ is readily calculable [49,63]. Likewise $\mathcal{V}$ and $\mathcal{G}$ are readily calculable for the equations of magnetohydrodynamics (MHD) [37, 51].

Remark 2.7. For a notion of glancing at points of variable multiplicity, see [51].

\footnotetext{
${ }^{4}$ These cannot so far as we can see be prescribed globally analytically in the general multidimensional case as stated in [67].
} 
2.4. Limiting behavior as $\rho, \tau \rightarrow 0^{+}$. Define the matrices

$$
\mathcal{A}_{ \pm}(\tilde{\xi}, \lambda):=\left(\lambda I+i A^{\tilde{\xi}}\right)\left(A^{1}\right)_{ \pm}^{-1}
$$

conjugate to the hyperbolic initial-boundary-value symbol above, where $A^{\tilde{\xi}}:=$ $\sum_{j=2}^{d} \xi_{j} A^{j}$ and $A_{ \pm}^{j}:=A^{j}( \pm \infty)$. As a consequence of (H2), the stable/unstable subspaces of $\mathcal{A}_{ \pm}(\tilde{\xi}, \lambda)$ remain spectrally separated, hence vary analytically in $(\tilde{\xi}, \lambda)$ on the set $\mathbb{R}^{d-1} \times\{\Re \lambda>0\}[29]$; see $[49,67]$ for further discussion. We have the following extension to the boundary $\Re \lambda=0$.

Lemma 2.8. Local to any point $(\tilde{\xi}, \lambda)=(\tilde{\xi}, i \tau) \in \mathbb{R}^{d-1} \times\{\Re \lambda=0\}$ not belonging to the codimension-one set $\mathcal{V} \cup \mathcal{G}$, there exist analytic choices of bases $\left\{\mathcal{R}_{j}^{ \pm}(\tilde{\xi}, \lambda)\right\}$ spanning the stable (resp. unstable) subspaces of $\mathcal{A}_{ \pm}(\tilde{\xi}, \lambda)$ for $\Re \lambda>0$.

Proof. Clearly, the stable (resp. unstable) subspace of $\mathcal{A}_{ \pm}$at $\Re \lambda=0$ continues analytically into $\Re \lambda>0$, by spectral separation, and thus admits a locally analytic basis. It is sufficient, therefore, to consider pure imaginary eigenvalues of $\mathcal{A}_{ \pm}$.

Defining $i \xi_{1}=\alpha_{r}^{ \pm}(\tilde{\xi}, \tau)$ as above, $\xi_{1}$ real, with $\tau=-a_{r}^{ \pm}(\xi)$ we find that the genuine eigenspace of $\mathcal{A}$ associated to eigenvalue $\alpha_{r}$ is $A^{1}$ times the total eigenspace (recall the semisimplicity assumption of (H2)) of $A^{\xi}$ associated with $a_{r}$, hence varies analytically so long as $(\tilde{\xi}, i \tau) \notin \mathcal{V}$. On the other hand, the condition $(\tilde{\xi}, i \tau) \notin \mathcal{G}$ is readily seen (see, e.g., $[49,62,67])$ to preclude a nontrivial Jordan block for $\mathcal{A}$, so that the total eigenspace of $\mathcal{A}$ is equal to the genuine eigenspace and thus varies analytically. It therefore admits a locally analytic basis.

Finally, observing that $\Re \alpha_{r}=\Im \xi_{1}=0$ by (H2) implies $a_{r}$ is real, hence $\lambda$ is pure imaginary, we see that $\Re \alpha_{r}$ is of constant sign on $\Re \lambda>0$. Thus, for $\Re \lambda>0$, the stable (resp. unstable) subspace of $\mathcal{A}_{ \pm}$is the direct sum of eigenspaces associated with all $\alpha_{r}$ with $\Re \alpha_{r}<0$ (resp. $>0$ ), hence by the foregoing discussion admits an analytic bases as claimed.

We can now state the following key result describing the structure of bases $w_{j}^{ \pm}$in the low-frequency, or "hyperbolic", limit $\rho \rightarrow 0^{+}$.

Lemma 2.9. For any $\left(\tilde{\xi}_{0}, \lambda_{0}\right) \in \mathbb{R}^{d-1} \times\left\{\Re \lambda_{0} \geq 0\right\}$ not in $\mathcal{V} \cup \mathcal{G}, D_{\tilde{\xi}, \lambda}(\rho)$ and also its component factors $w_{j}^{ \pm}$in (2.4) has a unique (jointly) analytic extension onto a neighborhood of $(\tilde{\xi}, \lambda, \rho)=\left(\tilde{\xi}_{0}, \lambda_{0}, 0\right)$. Moreover, $w_{j}^{ \pm}$may be chosen at $(\tilde{\xi}, \lambda, \rho)=\left(\tilde{\xi}_{0}, \lambda_{0}, 0\right)$, to satisfy the linearized traveling-wave equation

$$
\left(B^{11} w^{\prime}\right)^{\prime}=\left(A^{1} w\right)^{\prime}
$$

with boundary conditions

$$
\begin{array}{r}
w_{1}^{+}(+\infty)=\cdots=w_{i_{+}}^{+}(+\infty)=0 \\
w_{2 n-i_{-}+1}^{-}(-\infty)=\cdots=w_{2 n}^{-}(-\infty)=0
\end{array}
$$


and

$$
\begin{aligned}
& w_{j}^{+}(+\infty)=\left(A_{+}^{1}\right)^{-1} \mathcal{R}_{j}^{+}\left(\tilde{\xi}_{0}, \lambda_{0}\right), \quad j=i_{+}+1, \ldots, n, \\
& w_{n+j}^{-}(-\infty)=\left(A_{-}^{1}\right)^{-1} \mathcal{R}_{j}^{-}\left(\tilde{\xi}_{0}, \lambda_{0}\right), \quad j=1, \ldots, n-i_{-},
\end{aligned}
$$

$\mathcal{R}_{j}^{ \pm}$as defined in Lemma 2.8, where $i_{+}$denotes the number of negative eigenvalues of $A_{+}^{1}$, and $i_{-}$the number of positive eigenvalues of $A_{-}^{1}$. In addition, $w_{1}^{+}, \ldots, w_{i_{+}}^{+}$and $w_{2 n-i_{-}+1}^{-}, \ldots, w_{2 n}^{-}$may be chosen independent of $(\tilde{\xi}, \lambda)$.

Proof. Here, again, the essential point is to establish behavior of the limiting constant-coefficient equations as $x_{1} \rightarrow \pm \infty$, now written in polar coordinates, for $\rho, \tau \rightarrow 0^{+}$. At $\rho=0$, these reduce to $A_{ \pm}^{1} w^{\prime}=B_{ \pm}^{11} w^{\prime \prime}$, hence have a center subspace of dimension $n$, consisting of constant solutions, and stable and unstable subspaces of dimensions summing to $n$. The latter evidently continue analytically, by spectral separation. Projecting onto the (also analytic) continuation of the center subspace, we find after a routine calculation that the former correspond to stable and unstable subspaces of $\rho \mathcal{A}_{ \pm}+O\left(\rho^{2}\right)$, hence continue analytically by an argument quite similar to that of Lemma 2.8. Specifically, fixing $\rho=0$ and varying $\tau=\Re \lambda$ to the side $\tau>0$, we find by Lemma 2.8 that each of the eigenvalues $\rho \alpha_{j}^{ \pm}$of $\rho \mathcal{A}_{ \pm}+O\left(\rho^{2}\right)$ bifurcating from a single eigenvalue $\alpha_{j}^{ \pm}$of $\mathcal{A}_{ \pm}$must have the same sign for $\tau>0$, and thus for $\rho \geq 0, \tau \geq 0,(\rho, \tau) \neq(0,0)$. Noting that the corresponding total eigenspaces vary analytically, by spectral separation, we are done. For details, see [67].

\section{Evans function computations}

We are now ready to carry out the desired Evans function computations.

3.1. The low-frequency limit. For later use, we first review the proof of expansion $(1.9),(1.10)$ in the simple, analytic case $(\tilde{\xi}, \lambda) \notin \mathcal{V} \cup \mathcal{G}$. Note that the conclusion is somewhat stronger in this case, with quadratic order error term. For discussion of the case $(\tilde{\xi}, \lambda) \notin \mathcal{V}$, see [23-25, 51,62-64]; for the general case, see $[26]$.

Proposition 3.1 ( $[62,67])$. For a Lax shock satisfying $(\mathrm{H} 0)-(\mathrm{H} 4)$ and any pair $(\tilde{\xi}, \lambda) \in \mathbb{R}^{d-1} \times\{\Re \lambda \geq 0\}$ not in $\mathcal{V} \cup \mathcal{G}$

$$
D(\tilde{\xi}, \lambda)=\gamma \Delta(\tilde{\xi}, \lambda)+\mathcal{O}\left(|(\tilde{\xi}, \lambda)|^{2}\right),
$$

where $\gamma$ is a constant measuring transversality of the connection $\bar{u}(\cdot)$ (hence nonzero, by $(\mathrm{H} 4)), \mathcal{O}(\cdot)$ is uniform for $|(\tilde{\xi}, \lambda)|$ bounded, and $\Delta(\cdot, \cdot)$ is the Lopatinski determinant defined in (1.7), or, equivalently, $D_{\tilde{\xi}, \lambda}(0)=0$ and

$$
\frac{\partial}{\partial_{\rho}} D_{\tilde{\xi}, \lambda}(0)=\gamma \Delta(\tilde{\xi}, \lambda)
$$


Remark 3.2. Similar, but less explicit formulae hold for nonclassical over- or undercompressive shocks; see $[3,6,62,67]$.

Proof. Rewriting the generalized eigenvalue equation (2.3) in polar coordinates, we have

$$
\begin{aligned}
0=L_{\rho} w:= & \left(B^{11} w^{\prime}\right)^{\prime}-\left(A^{1} w\right)^{\prime}+i \rho \sum_{j \neq 1} B^{j 1} \xi_{j} w^{\prime}+i \rho\left(\sum_{k \neq 1} B^{1 k} \xi_{k} w\right)^{\prime} \\
& -i \rho \sum_{j \neq 1} A^{j} \xi_{j} w-\rho \lambda w-\rho^{2} \sum_{j \neq 1, k \neq 1} B^{j k} \xi_{j} \xi_{k} w .
\end{aligned}
$$

Recall that the rows of the Evans function determinant are made up of solutions $w_{j}^{ \pm}(\tilde{\xi}, \lambda, \rho)$, with boundary conditions $(2.6)-(2.7)$ at $\rho=0$. Note as usual that $L_{0} w=\left(B^{11} w^{\prime}\right)^{\prime}-\left(A^{1} w\right)^{\prime}$ reduces to the one-dimensional linearized operator about the wave.

The variation $\bar{u}^{\prime}$ satisfies (2.5) and decays exponentially at both $\pm \infty$, hence belongs to both of the fast-decaying manifolds

$$
\operatorname{span}\left\{w_{1}^{+}, \ldots, w_{i_{+}}^{+}\right\} \text {and } \operatorname{span}\left\{w_{2 n-i_{-}+1}^{-}, \ldots, w_{2 n}^{-}\right\}
$$

for $\rho=0$, all $(\tilde{\xi}, \lambda)$ (and indeed lies in the kernel of $L_{\tilde{\xi}}$ for all $\tilde{\xi}$ ). Without loss of generality, therefore, normalize $w_{1}^{+}=w_{2 n}^{-}=\bar{u}^{\prime}$ at $\rho=0$, for all $(\tilde{\xi}, \lambda)$.

With this choice, $D_{\tilde{\xi}, \lambda}(0)=0$ follows immediately from definition (2.4). Likewise, we find, applying the Leibnitz rule and performing elementary matrix manipulations, that

$$
\begin{aligned}
& \frac{\partial}{\partial \rho} D(0)=\operatorname{det}\left(\begin{array}{lll}
\frac{\partial}{\partial \rho} w_{1}^{+} & \cdots & w_{2 n}^{-} \\
\frac{\partial}{\partial \rho} w_{1}^{+\prime} & \cdots & w_{2 n}^{-\prime}
\end{array}\right)_{\left.\right|_{x_{1}=0}} \\
& +\operatorname{det}\left(\begin{array}{cccc}
w_{1}^{+} & \frac{\partial}{\partial \rho} w_{2}^{+} & \cdots & w_{2 n}^{-} \\
w_{1}^{+\prime} & \frac{\partial}{\partial \rho} w_{2}^{+\prime} & \cdots & w_{2 n}^{-\prime}
\end{array}\right)_{\mid x_{1}=0}+\cdots \\
& +\operatorname{det}\left(\begin{array}{lll}
w_{1}^{+} & \cdots & \frac{\partial}{\partial \rho} w_{2 n}^{-} \\
w_{1}^{+\prime} & \cdots & \frac{\partial}{\partial \rho} w_{2 n}^{-\prime}
\end{array}\right)_{\left.\right|_{x_{1}=0}} \\
& =\operatorname{det}\left(\begin{array}{lll}
\frac{\partial}{\partial \rho} w_{1}^{+} & \cdots & w_{2 n}^{-} \\
\frac{\partial}{\partial \rho} w_{1}^{+\prime} & \cdots & w_{2 n}^{-\prime}
\end{array}\right)_{\left.\right|_{x_{1}=0}}+0+\cdots+0 \\
& +\operatorname{det}\left(\begin{array}{ccc}
w_{1}^{+} & \cdots & \frac{\partial}{\partial \rho} w_{2 n}^{-} \\
w_{1}^{+\prime} & \cdots & \frac{\partial}{\partial \rho} w_{2 n}^{-\prime}
\end{array}\right)_{\mid x_{1}=0} \\
& =\operatorname{det}\left(\begin{array}{cccc}
w_{1}^{+} & \cdots & w_{2 n-1}^{-}, & \left(y^{-}-y^{+}\right) \\
w_{1}^{+\prime} & \cdots & w_{2 n-1}^{-} & \left(y^{-}-y^{+}\right)^{\prime}
\end{array}\right)_{\left.\right|_{x_{1}=0}},
\end{aligned}
$$


where $y^{+}:=\frac{\partial}{\partial \rho} w_{1}^{+}$and $y^{-}:=\frac{\partial}{\partial \rho} w_{2 n}^{-}$at $\rho=0$.

To evaluate this quantity, recall that $w=w_{j}^{ \pm}$satisfy $(2.5)$ at $\rho=0$, with boundary conditions (2.6) prescribed at $+\infty,-\infty$ respectively. Integrating $(2.5)$ from $+\infty /-\infty$ to $x_{1}$, we thus obtain the relation

$$
B^{11} w^{\prime}=A^{1} w-A^{1} w_{\left.\right|_{ \pm \infty}}, \quad \text { or } \quad B^{11} w^{\prime}-A^{1} w=-A^{1} w_{\left.\right|_{ \pm \infty}} .
$$

Referring to (2.6)-(2.7), we find that

$$
B^{11} w_{j}^{ \pm^{\prime}}-A^{1} w_{j}^{ \pm}=0
$$

for decaying modes $w_{1}^{+}, \ldots, w_{i_{+}}^{+}$and $w_{2 n-i_{-}+1}^{-}, \ldots, w_{2 n}^{-}$, while, for the asymptotically constant modes,

$$
\begin{aligned}
B^{11} w_{j}^{+\prime}-A^{1} w_{j}^{+} & =\mathcal{R}_{j}^{+}(\tilde{\xi}, \lambda), & & j=i_{+}+1, \ldots, n \\
B^{11} w_{n+j}^{-}{ }^{\prime}-A^{1} w_{n+j}^{-} & =\mathcal{R}_{j}^{-}(\tilde{\xi}, \lambda), & & j=1, \ldots, n-i_{-} .
\end{aligned}
$$

Differentiating (3.2), we find, further, that derivatives $y_{j}^{ \pm}:=\frac{\partial}{\partial \rho} w_{j}^{ \pm}$satisfy the first order variational equations

$$
\begin{aligned}
L_{\rho} y= & -\left[\frac{\partial}{\partial \rho} L_{\rho}\right] w \\
= & -i \sum_{j \neq 1} B^{j 1} \xi_{j} w^{ \pm \prime}-i\left(\sum_{k \neq 1} B^{1 k} \xi_{k} w^{ \pm}\right)^{\prime} \\
& +i \sum_{j \neq 1} A^{j} \xi_{j} w^{ \pm}+\lambda w^{ \pm}+2 \rho i \sum_{j \neq 1, k \neq 1} B^{j k} \xi_{j} \xi_{k} w
\end{aligned}
$$

with boundary conditions

$$
y^{+}(+\infty)=y^{-}(-\infty)=0 .
$$

Here, $w^{ \pm}$denote $w_{1}^{+}, w_{2 n}^{-}$respectively. Recalling that, for $\rho=0, w_{1}^{+}=w_{2 n}^{-}=\bar{u}^{\prime}$, and using the definition of $A^{j}$ in (2.2) to express $A^{j} \bar{u}^{\prime}-B^{j 1} \xi_{j} \bar{u}^{\prime \prime}$ as a perfect derivative, $f^{j}(\bar{u})^{\prime}-\left(B^{j 1}(\bar{u}) \bar{u}^{\prime}\right)^{\prime}$, we obtain, integrating (3.6) from $+\infty /-\infty$, respectively, to $x_{1}$, the relations

$$
\begin{aligned}
B^{11} y^{ \pm^{\prime}}-A^{1} y^{ \pm}= & i f^{\tilde{\xi}}(\bar{u})-i B^{1 \tilde{\xi}}(\bar{u}) \bar{u}^{\prime}-i B^{\tilde{\xi} 1}(\bar{u}) \bar{u}^{\prime}+\lambda \bar{u} \\
& -\left(i f^{\tilde{\xi}}\left(u_{ \pm}\right)+\lambda u_{ \pm}\right)
\end{aligned}
$$

and thus

$$
B^{11}\left(y^{-}-y^{+}\right)^{\prime}-A^{1}\left(y^{-}-y^{+}\right)=i\left[f^{\tilde{\xi}}(u)\right]+\lambda[u] .
$$

Combining (3.4)-(3.5) with (3.8), and recalling (3.3), we obtain by the row 
operation $B^{11} w^{\prime}-A^{1} w$ that

$$
\begin{aligned}
& \frac{\partial D}{\partial \rho}(0)=\frac{1}{\operatorname{det}\left(B^{11}\right)} \times \\
& \left|\begin{array}{ccccccccccccc}
w_{1}^{+} & \cdots & w_{i_{+}}^{+} & * & \cdots & * & * & \cdots & * & w_{2 n-i_{-}+1}^{-} & \cdots & w_{2 n-1}^{-} & * \\
0 & \cdots & 0 & \mathcal{R}_{i_{+}+1}^{+} & \cdots & \mathcal{R}_{n}^{+} & \mathcal{R}_{1}^{-} & \cdots & \mathcal{R}_{n-i_{-}}^{-} & 0 & \cdots & 0 & \diamond
\end{array}\right|_{x_{1}=0}
\end{aligned}
$$

where $\diamond=i\left[f^{\tilde{\xi}}(u)\right]+\lambda[u]$. Performing $\left(n-i_{+}\right)\left(n-i_{-}\right)+\left(2 n-i_{-}-i_{+}\right)\left(i_{-}-\right.$ $1) \equiv n-i_{-} \bmod 2$ column transpositions (exchanging second and third column blocks, then moving both past the fourth), and recalling $p=n-i_{-}+1$, we obtain relation (3.1), with

$$
\gamma:=\operatorname{det}\left(B^{11}\right)^{-1}(-1)^{p} \operatorname{det}\left(w_{1}^{+} \cdots w_{i_{+}}^{+} w_{2 n-i_{-}+1}^{-} \cdots w_{2 n-1}^{-}\right)_{\left.\right|_{1}=0} .
$$

Noting that $w_{1}^{+}, \ldots, w_{i_{+}}^{+}$and $w_{2 n-i_{-}+1}^{-}, \ldots, w_{2 n}^{-}$satisfy the (reduced, i.e. first order) linearized traveling-wave ODE

$$
B^{11} w^{\prime}=A^{1} w
$$

and vanish at $+\infty,-\infty$ respectively, we find that they span the tangent manifold along $\bar{u}(\cdot)$ of the stable/unstable manifolds at $u_{+} / u_{-}$respectively, and, by the discussion in $\S 2$, that (3.9) is indeed a transversality coefficient for the travelingwave ODE.

3.2. Computing $\beta$. Finally, we carry out our main computation. Let $\Delta(\tilde{\xi}, i \tau)$ vanish for some real, nontrivial $\tilde{\xi}, \tau$. Along with (H0)-(H4), assume also

(H5) $\mathcal{R}_{1}^{-}, \ldots, \mathcal{R}_{p-1}^{-}, \mathcal{R}_{p+1}^{+}, \ldots, \mathcal{R}_{n}^{+}$are independent at $(\tilde{\xi}, i \tau)$.

(H6) $(\tilde{\xi}, i \tau) \notin \mathcal{V} \cup \mathcal{G}$ (defined Section 2.3).

(H7) Each $\alpha_{j}$ is pure imaginary.

Hypothesis (H5), made for definiteness, holds always for extreme shocks ( $p=n$ or 1 , or equivalently $i_{-}$or $i_{+}=n$ ), and holds generically for intermediate shocks $(1<p<n)$. In general, it may be checked in the course of evaluating $\Delta$, $\Delta_{\lambda}$, at no extra computational cost. As mentioned earlier, $\mathcal{V} \cup \mathcal{G}$ is codimension one in $\{\Re \lambda=0\}$, so is also generically satisfied. In particular, since codimension one, these do not interfere with the problem of determining stability transitions as we move along a one-dimensional Hugoniot curve. Finally, as discussed in [4], condition (H7) ensures that the zero $(\tilde{\xi}, i \tau)$ lies in the "hyperbolic domain" for which the bases $\mathcal{R}_{j}$ may be chosen real at $\rho=0$, so that the instability is indeed of the "weakly real" type that persists on an open set in parameter space. This is always satisfied for $|\tilde{\xi}|$ sufficiently small with respect to $|\tau|$ (see [56]), and holds in particular for weak instabilities occurring in gas dynamics [42-44,62-64].

We establish the following result, of which Theorem 1.3 is a corollary. 
Proposition 3.3. Under the assumptions of Theorem 1.3, and taking without loss of generality $\mathcal{R}_{j}^{ \pm}$to be genuine eigenvectors of $\mathcal{A}_{ \pm}, \mathcal{A}_{ \pm} \mathcal{R}_{j}^{ \pm}=\alpha_{j}^{ \pm} \mathcal{R}_{j}^{ \pm}$, with $\mathcal{L}_{j}^{ \pm}$dual left eigenvectors, $\mathcal{L}_{j} \mathcal{A}_{ \pm}=\mathcal{L}_{j} \alpha_{j}^{ \pm}$, the conclusions of the Theorem hold with

$$
\begin{aligned}
\mathcal{B}= & \sum_{j<p} c_{j} \ell \cdot\left(B_{-}^{11} \alpha_{j}^{-} \mathcal{R}_{j}^{-}+\sum_{k \geq p} \frac{\mathcal{L}_{k}^{-} B_{-}\left(\tilde{\xi}, \alpha_{j}^{-}\right) \mathcal{R}_{j}^{-}}{\alpha_{k}^{-}} \mathcal{R}_{k}^{-}\right) \\
& +\sum_{j>p} c_{j} \ell \cdot\left(B_{+}^{11} \alpha_{j}^{+} \mathcal{R}_{j}^{+}+\sum_{k \leq p} \frac{\mathcal{L}_{k}^{+} B_{+}\left(\tilde{\xi}, \alpha_{j}^{+}\right) \mathcal{R}_{j}^{+}}{\alpha_{k}^{+}} \mathcal{R}_{k}^{+}\right),
\end{aligned}
$$

where $B_{ \pm}(\tilde{\xi}, \alpha):=\left(B^{11} \alpha^{2}+\sum_{j \neq 1}\left(B^{j 1}+B^{1 j}\right) \xi_{j} \alpha+\sum_{j, k \neq 1} B^{j k} \xi_{j} \xi_{k}\right)_{ \pm}$.

Proof. By Proposition 3.1, $\frac{\partial^{2}}{\partial_{\lambda} \partial_{\rho}} D(0)=\gamma \Delta_{\lambda}$. Thus, it suffices to establish

$$
\begin{aligned}
\left(\frac{\partial}{\partial_{\rho}}\right)^{2} D(0) & =\gamma\left[\left\langle l,-2\left[\frac{\partial}{\partial \rho} L_{\rho}\right]_{\rho=0} \tilde{y}-\left[\left(\frac{\partial}{\partial \rho}\right)^{2} L_{\rho}\right]_{\left.\right|_{\rho=0}} \bar{u}^{\prime}\right\rangle+\mathcal{B}\right] \\
& =\gamma\left[\left\langle l, 2\left(\lambda I+A^{\tilde{\xi}}-i B^{1 \tilde{\xi}} \partial_{x_{1}}-i \partial_{x_{1}} B^{\tilde{\xi} 1}\right) \tilde{y}+2 B^{\tilde{\xi} \tilde{\xi}} \bar{u}^{\prime}\right\rangle+\mathcal{B}\right],
\end{aligned}
$$

with $\mathcal{B}$ as in (3.10). We compute this second derivative by a calculation generalizing that of $[5]$ in the one-dimensional case.

Namely, following the general approach of $[19,20,32,66]$, we choose a convenient basis for the computation, consisting of a generalized Jordan chain. Specifically, we first choose again the normalization $w_{1}^{+}=w_{2 n}^{-}=\bar{u}^{\prime}$ at $\rho=0$. Next, we observe that, by the matrix manipulations carried out in the proof of Proposition 3.1 and the facts (by transversality assumption (H4)) that $\gamma \neq 0$ and the kernel of $B^{11} \partial_{x}-A^{1}$ is spanned by $\bar{u}^{\prime}$, the assumed relation $\frac{\partial}{\partial_{\rho}} D^{\tilde{\xi}, i \tau}(0)=0 \mathrm{im}-$ plies a linear dependence modulo $\bar{u}^{\prime}$ in the functions $w_{1}^{-}, \ldots, w_{p-1}^{-}, w_{p+1}^{+}, \ldots, w_{n}^{+}$ and $\frac{\partial}{\partial_{\rho}} w_{2 n}^{-}-\frac{\partial}{\partial \rho} w_{1}^{+}$, or

$$
\sum_{j=1}^{p-1} c_{j} w_{j}^{-}+\sum_{j=p+1}^{n} c_{j} w_{j}^{+}+c_{p} \bar{u}^{\prime}+\left(\frac{\partial}{\partial_{\rho}} w_{2 n}^{-}-\frac{\partial}{\partial \rho} w_{1}^{+}\right)=0
$$

where

$$
\sum_{j=1}^{p-1} c_{j} r_{j}^{-}+\sum_{j=p+1}^{n} c_{j} r_{j}^{+}+\left(i\left[f^{\tilde{\xi}}(u)\right]+\lambda[u]\right)=0 .
$$

Note that we have used (H6) in setting the coefficient of $\left(i\left[f^{\tilde{\xi}}(u)\right]+\lambda[u]\right)$ to unity in (3.12). Defining

$$
\tilde{w}_{2 n}^{-}:=w_{2 n}^{-}+\rho\left(c_{p} w_{2 n}^{-}+\sum_{j=1}^{p-1} c_{j} w_{j}^{-}\right), \quad \tilde{w}_{1}^{+}:=w_{1}^{+}-\rho \sum_{j=p+1}^{n} c_{j} w_{j}^{+},
$$


we thus have the generalized Jordan chain

$$
\tilde{w}_{1}^{+}=\tilde{w}_{2 n}^{-}=\bar{u}^{\prime}, \quad \frac{\partial}{\partial \rho} \tilde{w}_{1}^{+}=\frac{\partial}{\partial_{\rho}} \tilde{w}_{2 n}^{-} .
$$

We may substitute $\tilde{w}_{1}^{+}$and $\tilde{w}_{2 n}^{-}$for $w_{1}^{+}$and $w_{2 n}^{-}$without changing the value of $D$. With this choice of basis, we find readily, applying the Leibnitz rule and performing elementary matrix manipulations, similarly as in the proof of Proposition 3.1, that $\frac{\partial}{\partial_{\rho}} D(0)=0$, and

$$
\left(\frac{\partial}{\partial \rho}\right)^{2} D(0)=\operatorname{det}\left(\begin{array}{cccc}
w_{1}^{+} & \cdots & w_{2 n-1}^{-}, & \left(\tilde{z}^{-}-\tilde{z}^{+}\right) \\
w_{1}^{+\prime} & \cdots & w_{2 n-1}^{-} & \left(\tilde{z}^{-}-\tilde{z}^{+}\right)^{\prime}
\end{array}\right)_{\left.\right|_{x_{1}=0}}
$$

where $\tilde{z}^{+}:=\left(\frac{\partial}{\partial \rho}\right)^{2} \tilde{w}_{1}^{+}$and $z^{-}:=\left(\frac{\partial}{\partial \rho}\right)^{2} \tilde{w}_{2 n}^{-}$at $\rho=0$.

Now, second derivatives $\tilde{z}_{j}^{ \pm}:=\left(\frac{\partial}{\partial \rho}\right)^{2} \tilde{w}_{j}^{ \pm}$satisfy the second order variational equations

$$
\begin{aligned}
L_{\rho} \tilde{z}= & -2\left[\frac{\partial}{\partial \rho} L_{\rho}\right] \tilde{y}-\left[\left(\frac{\partial}{\partial \rho}\right)^{2} L_{\rho}\right] \tilde{w} \\
= & -2 i \sum_{j \neq 1} B^{j 1} \xi_{j} \tilde{y}^{\prime}-2 i\left(\sum_{k \neq 1} B^{1 k} \xi_{k} \tilde{y}\right)^{\prime} \\
& +2 i \sum_{j \neq 1} A^{j} \xi_{j} \tilde{y}+2 \lambda \tilde{y}+2 \rho \sum_{j \neq 1, k \neq 1} B^{j k} \xi_{j} \xi_{k} \tilde{y} \\
& +2 \sum_{j \neq 1, k \neq 1} B^{j k} \xi_{j} \xi_{k} \tilde{w},
\end{aligned}
$$

where $\tilde{y}:=\frac{\partial}{\partial \rho} \tilde{w}$, hence, setting $\rho=0$ and recalling $(3.14),\left(B^{11}\left(\tilde{z}^{-}-\tilde{z}^{+}\right)^{\prime}-\right.$ $\left.A^{1}\left(\tilde{z}^{-}-\tilde{z}^{-}\right)\right)^{\prime}=L_{0}\left(\tilde{z}^{-}-\tilde{z}^{+}\right)=0$, or $B^{11}\left(\tilde{z}^{-}-\tilde{z}^{+}\right)^{\prime}-A^{1}\left(\tilde{z}^{-}-\tilde{z}^{-}\right) \equiv I$ for some constant $I$.

Recalling again (3.4), (3.5), and performing the same row operation $B^{11} w^{\prime}-$ $A^{1} w$ used in the proof of Proposition 3.1, we thus obtain

$$
\left(\frac{\partial}{\partial_{\rho}}\right)^{2} D(0)=\gamma \operatorname{det}\left(\begin{array}{lllllll}
\mathcal{R}_{1}^{-} & \cdots & \mathcal{R}_{p-1}^{-} & \mathcal{R}_{p+1}^{+} & \cdots & \mathcal{R}_{n}^{+} & I)=\gamma l \cdot I,
\end{array}\right.
$$

where $l$ is defined as in (1.13). To evaluate $l \cdot I$, observe, from (3.15) and (3.14), that

$$
\begin{aligned}
L_{0} \tilde{z}^{ \pm}= & \left(B^{11}\left(\tilde{z}^{ \pm}\right)^{\prime}-A^{1}\left(\tilde{z}^{ \pm}\right)\right)^{\prime} \\
= & -2 i \sum_{j \neq 1} B^{j 1} \xi_{j} \tilde{y}^{\prime}-2 i\left(\sum_{k \neq 1} B^{1 k} \xi_{k} \tilde{y}\right)^{\prime} \\
& +2\left(\lambda+i A^{\tilde{\xi}}\right) \tilde{y}+2 \sum_{j \neq 1, k \neq 1} B^{j k} \xi_{j} \xi_{k} \bar{u}^{\prime} .
\end{aligned}
$$


By the Fundamental Theorem of Calculus, therefore,

$$
\begin{aligned}
l \cdot I= & \left.l \cdot\left(M_{0} \tilde{z}^{-}-M_{0} \tilde{z}^{+}\right)\right|_{x=0} \\
= & \int_{-\infty}^{+\infty} l \cdot L_{0} \tilde{z}^{ \pm} d x_{1}+\left.l \cdot L_{0} \tilde{z}^{+}\right|_{+\infty}-\left.l \cdot L_{0} \tilde{z}^{-}\right|_{-\infty} \\
= & \int_{-\infty}^{+\infty} l \cdot\left(-2 i \sum_{j \neq 1} B^{j 1} \xi_{j} \tilde{y}^{\prime}-2 i\left(\sum_{k \neq 1} B^{1 k} \xi_{k} \tilde{y}\right)^{\prime}\right. \\
& \left.+2\left(\lambda+i A^{\tilde{\xi}}\right) \tilde{y}+2 \sum_{j \neq 1, k \neq 1} B^{j k} \xi_{j} \xi_{k} \bar{u}^{\prime}\right) d x_{1} \\
& +\left.l \cdot\left(B^{11}\left(\tilde{z}^{+}\right)^{\prime}-A^{1} \tilde{z}^{+}\right)\right|_{+\infty}-\left.l \cdot\left(B^{11}\left(\tilde{z}^{-}\right)^{\prime}-A^{1} \tilde{z}^{-}\right)\right|_{-\infty}
\end{aligned}
$$

so long as the righthand side converges, where $L_{0}=: \partial_{x_{1}} M_{0}$.

Differentiating (3.13) with respect to $\rho$, we obtain

$$
\tilde{y}_{2 n}^{-}=y_{2 n}^{-}+\left(c_{p} w_{2 n}^{-}+\sum_{j=1}^{p-1} c_{j} w_{j}^{-}\right), \quad \tilde{y}_{1}^{+}:=y_{1}^{+}-\sum_{j=p+1}^{n} c_{j} w_{j}^{+},
$$

and

$$
\tilde{z}_{2 n}^{-}=z_{2 n}^{-}+\left(c_{p} y_{2 n}^{-}+\sum_{j=1}^{p-1} c_{j} y_{j}^{-}\right), \quad \tilde{z}_{1}^{+}:=z_{1}^{+}-\sum_{j=p+1}^{n} c_{j} y_{j}^{+}
$$

where $y_{j}^{ \pm}$and $z_{j}^{ \pm}$decay exponentially as $x_{1} \rightarrow+\infty$ (resp. $\left.-\infty\right)$, as do $\tilde{w}_{2 n}^{-}=$ $\tilde{w}_{1}^{+}=\bar{u}_{\tilde{s}}^{\prime}$ and $\left(\tilde{y}_{j}^{ \pm}\right)^{\prime}$. Recalling (2.6)-(2.7), the definition of $r_{j}^{ \pm}$as eigenvectors of $\left(\lambda+i A^{\tilde{\xi}}\right)\left(A^{1}\right)^{-1}$, and the fact that $l$ was chosen orthogonal to all $r_{j}^{ \pm}$appearing in (3.17), we find that the integral on the righthand side of (3.16) indeed converges, though

$\int_{-\infty}^{+\infty}\left(-2 i \sum_{j \neq 1} B^{j 1} \xi_{j} \tilde{y}^{\prime}-2 i\left(\sum_{k \neq 1} B^{1 k} \xi_{k} \tilde{y}\right)^{\prime}+2\left(\lambda+i A^{\tilde{\xi}}\right) \tilde{y}+2 \sum_{j \neq 1, k \neq 1} B^{j k} \xi_{j} \xi_{k} \tilde{w}\right) d x_{1}$

may not.

A more delicate issue is the convergence of boundary terms in $\tilde{z}^{ \pm}$, which amounts, by (3.18), to the assignment of boundary values for $y_{j}^{ \pm}$at $\rho=0$ for the slow-decaying modes $w_{j}^{ \pm}$appearing in formulae (3.13), (3.17). As it will play an important role in the calculations, we carry this out separately, in some detail.

Lemma 3.4. Assume $(\mathrm{H} 0)-(\mathrm{H} 7)$. For $\rho=0$, let $w_{j}^{+} \sim\left(A_{+}^{1}\right)^{-1} \mathcal{R}_{j}^{+}$as $x_{1} \rightarrow+\infty$, with

$$
\mathcal{A}_{+} \mathcal{R}_{j}^{+}=\alpha_{j} \mathcal{R}_{j}^{+}, \quad \mathcal{L}_{j}^{+} \mathcal{A}_{+}=\mathcal{L}_{j}^{+} \alpha_{j}, \quad \mathcal{L}_{j}^{+} \mathcal{R}_{j}^{+}=\delta_{j}^{k}
$$


at $(\tilde{\xi}, \lambda) \notin \mathcal{V} \cup \mathcal{C}$, where $\mathcal{A}_{+}=\left(\lambda+i A^{\tilde{\xi}}\right)\left(A^{1}\right)_{+}^{-1}$ as in (1.8) and $\delta_{j}^{k}$ is the Kronecker delta function. Then, the corresponding variational boundary conditions at $\rho=0$ are

$$
\begin{aligned}
y_{j}^{+} & \sim-x \alpha_{j}\left(A_{+}^{1}\right)^{-1} \mathcal{R}_{j}^{+}+\left(A_{+}^{1}\right)^{-1} \frac{\partial}{\partial_{\rho}}\left(\mathcal{R}_{j}^{+}\right), \\
\left(y_{j}^{+}\right)^{\prime} & \sim-\alpha_{j}\left(A_{+}^{1}\right)^{-1} \mathcal{R}_{j}^{+},
\end{aligned}
$$

where all error terms are exponentially decaying and

$$
\begin{aligned}
\frac{\partial}{\partial_{\rho}} \mathcal{R}_{j}^{+} & =\sum_{\alpha_{k}^{+} \neq \alpha_{j}^{+}} \frac{\mathcal{L}_{k}^{+} B\left(\tilde{\xi}, \alpha_{j}\right) \mathcal{R}_{j}^{+}}{\alpha_{k}} \mathcal{R}_{k}^{+}+\sum_{\alpha_{k}^{+}=\alpha_{j}^{+}} d_{k} \mathcal{R}_{k}^{+}, \\
B(\tilde{\xi}, \alpha) & :=B_{+}^{11} \alpha^{2}+\sum_{j \neq 1}\left(B_{+}^{j 1}+B_{+}^{1 j}\right) \xi_{j} \alpha+\sum_{j, k \neq 1} B_{+}^{j k} \xi_{j} \xi_{k} .
\end{aligned}
$$

A symmetric result holds for modes $w_{j}^{-} \sim\left(A_{-}^{1}\right)^{-1} \mathcal{R}_{j}^{-}$as $x_{1} \rightarrow-\infty$.

Proof. Again, it is sufficient to show the corresponding result for the limiting, constant-coefficient equations as $x_{1} \rightarrow \pm \infty$, the variable-coefficient result then following by asymptotic ODE theory (the "conjugation lemma" [50]).

Let us first consider the simpler, strictly hyperbolic case, for which, away from $\mathcal{G}$, the eigenvalues of $\mathcal{A}_{ \pm}$are simple, and we can choose slow modes analytically as pure exponential modes $w\left(\tilde{\xi}, \lambda, \rho, x_{1}\right)=e^{\mu x_{1}} v(\tilde{\xi}, \lambda, \rho)$. Substituting this Ansatz into the frozen-coefficient version of (3.2), we obtain

$$
\begin{aligned}
0= & \mu^{2} B_{+}^{11} v-\mu A_{+}^{1} v+i \mu \rho \sum_{j \neq 1} B_{+}^{j 1} \xi_{j} v+i \mu \rho \sum_{k \neq 1} B_{+}^{1 k} \xi_{k} v \\
& -i \rho \sum_{j \neq 1} A_{+}^{j} \xi_{j} v-\rho \lambda v-\rho^{2} \sum_{j \neq 1, k \neq 1} B_{+}^{j k} \xi_{j} \xi_{k} v
\end{aligned}
$$

from which we readily find that slow modes satisfy $\mu \sim-\rho \alpha$ as $\rho \rightarrow 0$, so that $\mu_{\rho}=-\alpha$ at $\rho=0$ for all modes associated with a given $\alpha_{j}$, whereupon (3.19) follow by the product rule. In the general (nonstrictly hyperbolic) case, away from $\mathcal{V} \cup \mathcal{G}$, the eigenvalues $\alpha$ of $\mathcal{A}_{+}$occur in constant-multiplicity blocks, and $w\left(\tilde{\xi}, \lambda, \rho, x_{1}\right)=e^{m x_{1}} v(\tilde{\xi}, \lambda, \rho)$, with $m=-\rho \alpha I_{K}+O\left(\rho^{2}\right)$ where $K$ is the multiplicity of the block, from which we obtain the same result.

To make the calculation (3.20), we first note that, modulo the arbitrary term $\sum_{\alpha_{k}=\alpha_{j}} d_{k} \mathcal{R}_{k}^{+},\left.\frac{\partial}{\partial_{\rho}}\right|_{\rho=0}$ is independent under $C^{1}$ coordinate changes, being completely determined by the values of $\mathcal{R}_{j}^{+}$at $\rho=0$. Thus, we are free to choose modes $w_{j}^{+}$as pure exponentials $w\left(\tilde{\xi}, \lambda, \rho, x_{1}\right)=e^{\mu x_{1}} v(\tilde{\xi}, \lambda, \rho)$ so long as the eigenvectors $\mathcal{R}:=A^{1} v$ are $C^{1}$ with respect to $\rho$ : in particular, in the strictly hyperbolic case. 
More generally, substituting $\mu_{j}^{+}=:-\rho \alpha_{j}^{+}, A^{1} v_{j}^{+}=: \mathcal{R}_{j}^{+}$into (3.21) and dividing out a factor of $\rho$, we obtain the reduced (slow) equation

$$
\left(\mathcal{A}_{+}+\rho B\left(\tilde{\xi}, \alpha_{j}^{+}\right)-\alpha_{j}^{+}\right) \mathcal{R}_{j}^{+}=0
$$

with $B(\tilde{\xi}, \alpha)$ as in $(3.20)$, from which we see that $\alpha_{j}^{+}, \mathcal{R}_{j}^{+}$are generically $C^{1}$ as the values of $B_{+}^{j k}$ are varied. Thus, it is sufficient to establish (3.20) in this case, with the general result following by continuity. Assume, therefore, that $\alpha_{j}^{+}, \mathcal{R}_{j}^{+}$are $C^{1}$ solutions of (3.22). Differentiating (3.22) with respect to $\rho$ at $\rho=0$, taking products with $\mathcal{L}_{k}^{+}$for all $\alpha_{k}^{+} \neq \alpha_{j}^{+}$, and rearranging using the normalization $\mathcal{L}_{k}^{+} \mathcal{R}_{j}^{+}=\delta_{j}^{k}$, we readily obtain (3.20).

See [5] for a similar calculation in the one-dimensional case.

From (3.19), and the fact that $\ell$ is orthogonal to all $\mathcal{R}_{j}^{ \pm}$under consideration (i.e., the ones occurring as limits of the slow decaying modes appearing in the Evans function), we readily obtain

$$
\begin{aligned}
\mathcal{B} & :=\left.l \cdot\left(B^{11}\left(\tilde{z}^{+}\right)^{\prime}-A^{1} \tilde{z}^{+}\right)\right|_{+\infty}-\left.l \cdot\left(B^{11}\left(\tilde{z}^{-}\right)^{\prime}-A^{1} \tilde{z}^{-}\right)\right|_{-\infty} \\
& =\sum_{j \neq p} c_{j} \ell \cdot\left(B^{11} \alpha_{j} \mathcal{R}_{j}^{ \pm}+\frac{\partial}{\partial_{\rho}}\left(\mathcal{R}_{j}^{ \pm}\right)\right)
\end{aligned}
$$

whence (3.16) yields (3.11), (3.10) as claimed, where $c_{j}$ are as in (3.12), (3.19), and $\frac{\partial}{\partial_{\rho}} \mathcal{R}_{j}^{ \pm}$as in $(3.20)$.

Remark 3.5. As noted in the one-dimensional case [5], the calculations somewhat simplify in the case of an extreme shock, $p=n$ (resp. $p=1$ ), for which $\ell=\mathcal{L}_{1}^{+}$(resp. $\ell=\mathcal{L}_{n}^{-}$); in particular, this induces considerable cancellation in the expression $(3.10)$ for $\mathcal{B}$. In the one-dimensional case, $B(\tilde{\xi}, \alpha)=B^{11} \alpha^{2}$, leading to still further cancellation.

\section{Numerical approximation of $\tilde{y}$}

The computation of $\mathcal{B}$ or $\Delta$ amounts (see (3.10), (1.7)) to calculation of eigenvectors and eigenvalues of $\mathcal{A}_{ \pm}$, a standard numerical problem. In the case of gas dynamics, they may be computed in closed form. Thus, the computation of $\beta$ reduces (see (1.12)) to the solution of (1.14) for $\tilde{y}$, a rather nonstandard problem due to the absence of spectral gap for operator $L_{0}$, reflected at this level by the presence of nondecaying solutions as $x_{1} \rightarrow \pm \infty$. We may easily remove this degeneracy, however, by integrating the equations from $x_{1}=-\infty$ 
to $x_{1}$, and taking advantage of the facts (see (3.17), Section 3.2, and (2.6)) that

$$
\begin{array}{ll}
\lim _{z \rightarrow-\infty} \tilde{y}(z)=\left(A_{-}^{1}\right)^{-1} \sum_{j=1}^{p-1} c_{j} \mathcal{R}_{j}^{-}, & \lim _{z \rightarrow-\infty} \tilde{y}^{\prime}(z)=0 \\
\lim _{z \rightarrow+\infty} \tilde{y}(z)=\left(A_{+}^{1}\right)^{-1} \sum_{j=p+1}^{n} c_{j} \mathcal{R}_{j}^{+}, & \lim _{z \rightarrow+\infty} \tilde{y}^{\prime}(z)=0,
\end{array}
$$

$c_{j}$ determined by (3.12), hence

$$
\lim _{z \rightarrow-\infty}\left(B^{11} \tilde{y}^{\prime}-A^{1} \tilde{y}\right)(z)=-\sum_{j=1}^{p-1} c_{j} \mathcal{R}_{j}^{-},
$$

and (see (3.7)) that the righthand side of (1.14) is a perfect derivative, to obtain the first-order system

$$
\begin{aligned}
B^{11} \tilde{y}^{\prime}-A^{1} \tilde{y}=F:= & i f^{\tilde{\xi}}(\bar{u})-i B^{1 \tilde{\xi}}(\bar{u}) \bar{u}^{\prime}-i B^{\tilde{\xi} 1}(\bar{u}) \bar{u}^{\prime}+\lambda \bar{u} \\
& +\sum_{j=1}^{p-1} c_{j} \mathcal{R}_{j}^{-}-\left(i f^{\tilde{\xi}}\left(u_{-}\right)+\lambda u_{-}\right)
\end{aligned}
$$

with boundary conditions (4.1). Defining $\hat{y}:=\tilde{y}-y_{0}$, where

$$
y_{0}:=\left(\chi(z)\left(A_{-}^{1}\right)^{-1} \sum_{j=1}^{p-1} c_{j} \mathcal{R}_{j}^{-}+(1-\chi(z))\left(A_{+}^{1}\right)^{-1} \sum_{j=p+1}^{n} c_{j} \mathcal{R}_{j}^{+}\right)
$$

and

$$
\chi(z):=\frac{1+\tanh (z)}{2} \rightarrow \begin{cases}0, & z \rightarrow+\infty \\ 1, & z \rightarrow-\infty\end{cases}
$$

so that $\lim _{z \rightarrow \pm \infty} \hat{y}(z)=0$, we may convert (4.2), (4.1) to a boundary-value problem of the standard form discussed in, e.g. [54,55], namely

$$
B^{11} \tilde{y}^{\prime}-A^{1} \tilde{y}=\hat{F}:=F+\left(B^{11} y_{0}^{\prime}-A^{1} y_{0}\right),
$$

with "projective boundary conditions" at infinity,

$$
\lim _{z \rightarrow \pm \infty} \Pi_{ \pm} \hat{y}(z)=0
$$

where $\Pi_{ \pm}$are any projections that are isomorphisms on the subspaces of exponentially growing (resp. decaying) solutions of the limiting constant-coefficient equations $B_{+}^{11} y^{\prime}-A_{+}^{1} y=0$ (resp. $B_{-}^{11} y^{\prime}-A_{-}^{1} y=0$ ), augmented with a single boundary condition at $z=0$,

$$
\ell \cdot y=0
$$


to fix a unique solution (recall that $\left(B^{11} \partial_{x}-A^{1}\right)$ has one-dimensional kernel $\left.\operatorname{span}\left\{\bar{u}^{\prime}\right\}\right)$. As operator $B^{11} \partial_{x_{1}}-A^{1}$ has exponential dichotomies on $[0,+\infty)$ and $(-\infty, 0]$, the problem is numerically well-conditioned by standard considerations (see $[54,55]$ for details), and may be solved by any reasonable collocation method on a truncated domain $[-M, M]$, with $M>0$ sufficiently large. This completes our description of the numerical approximation of $\beta$.

Remark 4.1. In practice, one may solve (4.2) with inhomogeneous projective boundary conditions $\lim _{z \rightarrow \pm \infty} \Pi_{ \pm}\left(\tilde{y}(z)-\lim _{z \rightarrow \pm \infty} \tilde{y}(z)\right)=0$ at infinity and modified condition $\ell \cdot \tilde{y}(0)=0$ at $z=0$, so long as $M$ need not be too large. (Unlike $\hat{F}, F$ is nondecaying as $z \rightarrow \pm \infty$, and so there is a great deal of cancellation, with associated loss of significance, in the righthand side of (4.2) for large values of $|z|$.)

\section{References}

[1] Alexander, J., Gardner, R. and Jones, C. K. R. T., A topological invariant arising in the analysis of traveling waves. J. Reine Angew. Math. 410 (1990), $167-212$.

[2] Barmin, A. A. and Egorushkin, S. A., Stability of shock waves. Adv. Mech. 15 (1992)(1-2), 3 - 37 .

[3] Benzoni-Gavage, S., Linear stability of propagating phase boundaries in capillary fluids. Physica D 155 (2001)(3-4), 235 - 273.

[4] Benzoni-Gavage, S., Rousset, F., Serre, D. and Zumbrun, K., Generic types and transitions in hyperbolic initial-boundary-value problems. Proc. Roy. Soc. Edinburgh Sect. A 132 (2002), 1073 - 1104.

[5] Benzoni-Gavage, S., Serre, D. and Zumbrun, K., Alternate Evans functions and viscous shock waves. SIAM J. Math. Anal. 32 (2001), 929 - 962.

[6] Bertozzi, A., Münch, A., Shearer, M. and Zumbrun, K. , Stability of compressive and undercompressive thin film travelling waves. The dynamics of thin fluid films. European J. Appl. Math. 12 (2001), 253 - 291.

[7] Bridges,T. J., Derks, G. and Gottwald, G., Stability and instability of solitary waves of the fifth-order KdV equation: a numerical framework. Physica D 172 (2002), $190-216$.

[8] Brin, L., Numerical testing of the stability of viscous shock waves. Doctoral thesis. Bloomington (IN): Indiana University 1998.

[9] Brin, L., Numerical testing of the stability of viscous shock waves. Math. Comp. 70 (235) (2001), $1071-1088$.

[10] Brin, L. and Zumbrun, K., Analytically varying eigenvectors and the stability of viscous shock waves. Mat. Contemp. 22 (2002), $19-32$.

[11] Coulombel, J.-F., Stability of multidimensional undercompressive shock waves. Interfaces Free Bound. 5 (2003)(4), 367 - 390. 
[12] Coulombel, J.-F. and Secchi, P., Nonlinear compressible vortex sheets in two space dimensions. Ann. Sci. École Norm. Sup. 41 (2008)(1), 85-139.

[13] Erpenbeck, J. J., Stability of step shocks. Phys. Fluids 5 (1962)(10), 1181 1187.

[14] Evans, J. W., Nerve axon equations. I. Linear approximations. Indiana Univ. Math. J. 21 (1972), $877-885$.

[15] Evans, J. W., Nerve axon equations. II. Stability at rest. Indiana Univ. Math. J. 22 (1972), $75-90$.

[16] Evans, J. W., Nerve axon equations. III. Stability of the nerve impulse. Indiana Univ. Math. J. 22 (1972), 577 - 593.

[17] Evans, J.W., Nerve axon equations. IV. The stable and the unstable impulse. Indiana Univ. Math. J. 24 (1975), 1169 - 1190.

[18] Freistühler, H., A short note on the persistence of ideal shock waves. Arch. Math. (Basel) 64 (1995)(4), $344-352$.

[19] Gardner, R. and Jones, C. K. R. T., A stability index for steady state solutions of boundary value problems for parabolic systems. J. Diff. Eqs. 91 (1991)(2), $181-203$.

[20] Gardner, R. and Jones, C. K. R. T., Traveling waves of a perturbed diffusion equation arising in a phase field model. Indiana Univ. Math. J. 38 (1989)(4), $1197-1222$.

[21] Gardner, R. and Zumbrun, K., The gap Lemma and geometric criteria for instability of viscous shock profiles. Comm. Pure Appl. Math. 51 (1998)(7), $797-855$.

[22] Guès, O., Métivier, G., Williams, M. and Zumbrun, K., Multidimensional viscous shocks. I. Degenerate symmetrizers and long time stability. J. Amer. Math. Soc. 18 (2005), $61-120$.

[23] Guès, O., Métivier, G., Williams, M. and Zumbrun, K., Multidimensional viscous shocks. II: The small viscosity problem. Comm. Pure and Appl. Math. 57 (2004), $141-218$.

[24] Guès, O., Métivier, G., Williams, M. and Zumbrun, K., Existence and stability of multidimensional shock fronts in the vanishing viscosity limit. Arch. Rat. Mech. Anal. 175 (2005), 151 - 244.

[25] Guès, O., Métivier, G., Williams, M. and Zumbrun, K., Navier-Stokes regularization of multidimensional Euler shocks. Ann. Sci. École Norm. Sup. (4) 39 (2006), $75-175$.

[26] Guès, O., Métivier, G., Williams, M. and Zumbrun, K., Viscous Boundary Value Problems for Symmetric Systems with Variable Multiplicities. J. Diff. Eqs. 244 (2008)(2), 309 - 387.

[27] Guès, O., Métivier, G., Williams, M. and Zumbrun, K., Nonclassical multidimensional viscous and inviscid shocks. Duke Math. J. 142 (2008)(1), $1-110$.

[28] Henry, D., Geometric Theory of Semilinear Parabolic Equations. Lecture Notes Math. 840. Berlin: Springer 1981. 
[29] Hersh, R., Mixed problems in several variables. J. Math. Mech. 12 (1963), $317-334$.

[30] Humpherys, J. and Zumbrun, K., An efficient shooting algorithm for Evans function calculations in large systems. Physica D 220 (2006), 116 - 126.

[31] Jenssen, K., Lyng, G. and Williams, M., Equivalence of low-frequency stability conditions for multidimensional detonations in three models of combustion. Indiana Univ. Math. J. 54 (2005), 1 - 64.

[32] Kapitula, T., The Evans function and generalized Melnikov integrals. SIAM J. Math. Anal. 30 (1999)(2), 273 - 297.

[33] Kapitula, T. and Rubin, J., Existence and stability of standing hole solutions to complex Ginzburg-Landau equations. Nonlinearity 13 (2000), 77 - 112.

[34] Kapitula, T. and Sandstede, B., Stability of bright solitary-wave solutions to perturbed nonlinear Schrödinger equations. Physica D 124 (1998)(1-3), $58-103$.

[35] Kapitula, T. and Sandstede, B., Instability mechanism for bright solitary-wave solutions to the cubic-quintic Ginzburg-Landau equation. J. Opt. Soc. Amer. B Opt. Phys. 15 (1998)(11), 2757 - 2762.

[36] Kato, T., Perturbation Theory for Linear Operators. Berlin: Springer 1985.

[37] Kwon, B., Symbolic structure of the full MHD equations. Preprint 2006. Bloomington (IN): Indiana University.

[38] Li, Y. and Promislow, K., The mechanism of the polarizational mode instability in birefringent fiber optics. SIAM J. Math. Anal. 31 (2000)(6), $1351-1373$.

[39] Lin, X.-B., Generalized Rankine-Hugoniot condition and shock solutions for quasilinear hyperbolic systems. Special issue in celebration of Jack K. Hale's 70th birthday, Part 2 (Atlanta, GA/Lisbon, 1998). J. Diff. Eqs. 168 (2000)(2), $321-354$.

[40] Lyng, G. and Zumbrun, K., One-dimensional stability of viscous strong detonation waves. Arch. Ration. Mech. Anal. 173 (2004)(2), 213 - 277.

[41] Lyng, G. and Zumbrun, K., A stability index for detonation waves in Majda's model for reacting flow. Physica D 194 (2004)(1-2), 1 - 29.

[42] Majda, A., The Stability of Multidimensional Shock Fronts. Mem. Amer. Math. Soc. 43 (1983), no. 275.

[43] Majda, A., The Existence of Multidimensional Shock Fronts. Mem. Amer. Math. Soc. 41 (1983), no. 281.

[44] Majda, A., The Existence and Stability of Multidimensional Shock Fronts. Bull. Amer. Math. Soc. (N.S.) 4 (1981)(3), $342-344$.

[45] Majda, A. and Pego, R., Stable viscosity matrices for systems of conservation laws. J. Diff. Eqs. 56 (1985), 229 - 262.

[46] Majda, A. and Rosales, R., A theory for spontaneous formation of Mach stems in reacting shock fronts. I. The basic perturbation analysis. SIAM J. Appl. Math. 43 (1983)(6), 1310 - 1334. 
[47] Majda, A. and Rosales, R., A theory for spontaneous Mach-stem formation in reacting shock fronts. II. Steady-wave bifurcations and the evidence for breakdown. Stud. Appl. Math. 71 (1984)(2), $117-148$.

[48] Mascia, C. and Zumbrun, K., Pointwise Green function bounds for shock profiles with degenerate viscosity. Arch. Rat. Mech. Anal. 169 (2003), $177-263$.

[49] Métivier, G., Stability of multidimensional shocks. Advances in the Theory of Shock Waves. Progr. Nonlinear Diff. Eqs. Appl. 47. Boston (MA): Birkhäuser 2001, pp. $25-103$.

[50] Métivier, G. and Zumbrun, K., Large Viscous Boundary Layers for Noncharacteristic Nonlinear Hyperbolic Problems. Mem. Amer. Math. Soc. 175 (2005), no. 826.

[51] Métivier, G. and Zumbrun, K., Hyperbolic boundary value problems for symmetric systems with variable multiplicities. J. Diff. Eqs. 211 (2005), $61-134$.

[52] Mokrane, A., Problèmes mixtes hyperboliques nonlineaires. Thèse. Université de Rennes I, 1987.

[53] Pego, R. L. and Weinstein, M. I., Eigenvalues and instabilities of solitary waves. Philos. Trans. Roy. Soc. London Ser. A 340 (1992), $47-94$.

[54] Sandstede, B., Stability of travelling waves. In: Handbook of Dynamical Systems, Vol. 2. Amsterdam: North-Holland (2002), pp. 983 - 1055.

[55] Sandstede, B. and Scheel, A., Absolute and convective instabilities of waves on unbounded and large bounded domains. Physica D 145 (2000) 233-277.

[56] Serre, D., La transition vers l'instabilité pour les ondes de chocs multidimensionnelles. Trans. Amer. Math. Soc. 353 (2001), $5071-5093$.

[57] Serre, D., Systems of Conservation Laws. 1. Hyperbolicity, Entropies, Shock Waves. Cambridge: Cambridge Univ. Press 1999.

[58] Serre, D., Systems of 'Conservation Laws. 2. Geometric Structures, Oscillations, and Initial-Boundary Value Problems. Cambridge: Cambridge Univ. Press 2000.

[59] Texier, B. and Zumbrun, K., Relative Poincaré-Hopf bifurcation and galloping instability of traveling waves. Methods Appl. Anal. 12 (2005), 349 - 380.

[60] Texier, B. and Zumbrun, K., Galloping instability of viscous shock waves. To appear in: Physica D (2008).

[61] Wang, Y.-G. and Xin, Z., Stability and existence of multidimensional subsonic phase transitions. Acta Math. Appl. Sin. Engl. Ser. 19 (2003)(4), 529 - 558.

[62] Zumbrun, K., Stability of large amplitude shock waves of the compressible Navier-Stokes equation. Handbook of Fluid Dynamics Vol. III. Amsterdam: North-Holland 2004, pp. $311-533$.

[63] Zumbrun, K., Multidimensional stability of planar viscous shock waves. Advances in the theory of shock waves. In: Progr. Nonlinear Diff. Eqs. Appl. 47. Boston (MA): Birkhäuser 2001, pp. $307-516$. 
[64] Zumbrun, K., Planar stability criteria for viscous shock waves of systems with real viscosity. CIME Summer School Lecture Notes. Preprint 2004.

[65] Zumbrun, K., A sharp stability criterion for soliton-type propagating phase boundaries in Korteweg's model. Z. Anal. Anwendungen 27 (2008)(1), 11 - 30.

[66] Zumbrun, K. and Howard, P., Pointwise semigroup methods and stability of viscous shock waves. Indiana Univ. Math. J. 47 (1998), $741-871$.

[67] Zumbrun, K. and Serre, D., Viscous and inviscid stability of multidimensional planar shock fronts. Indiana Univ. Math. J. 48 (1999), 937 - 992.

Received September 17, 2006 